19 Revue d'histoire du XIXe siècle

Société d'histoire de la révolution de 1848 et des

révolutions du XIXe siècle

$57 \mid 2018$

Libido sciendi

\title{
Patrick BOUCHERON (dir.), Histoire mondiale de la
}

France

Raymond Huard

(2) OpenEdition

Journals

Édition électronique

URL : https://journals.openedition.org/rh19/6158

DOI : $10.4000 /$ rh 19.6158

ISSN : $1777-5329$

Éditeur

La Société de 1848

Édition imprimée

Date de publication : 26 décembre 2018

Pagination : 202-204

ISSN : 1265-1354

Référence électronique

Raymond Huard, «Patrick вoucheron (dir.), Histoire mondiale de la France », Revue d'histoire du XIXe siècle [En ligne], 57 | 2018, mis en ligne le 26 décembre 2018, consulté le 21 janvier 2022. URL : http:// journals.openedition.org/rh19/6158; DOI : https://doi.org/10.4000/rh19.6158

Ce document a été généré automatiquement le 21 janvier 2022.

Tous droits réservés 


\title{
Patrick BOUCHERON (dir.), Histoire mondiale de la France
}

\author{
Raymond Huard
}

\section{RÉFÉRENCE}

Patrick BOUCHERON (dir.), Histoire mondiale de la France, coordonné par Nicolas

Delalande, Florian Mazel, Yann Potin, Pierre Singaravélou, Paris, Seuil, 2017, 795 p., $29 €$.

1 Les historiens du XIX ${ }^{\mathrm{e}}$ siècle ne peuvent être indifférents à cette Histoire mondiale de la France dont la publication en 2017 a donné lieu à une large publicité et a constitué un événement historiographique, marqué par des polémiques. Notre propos vise simplement à examiner comment le XIX siècle apparaît dans cet ouvrage en tenant compte évidemment des intentions des auteurs du livre exposées avec beaucoup de clarté par Patrick Boucheron dans une « Ouverture » préalable. L'objectif était d'offrir à un large public "un livre innovant». Innovant en quoi? Non pas tellement par la rédaction qui devait être "vivante ", "accessible», ni par la forme qui serait celle " familière d'une collection de dates ", mais pour d'autres raisons. D'abord une intention «politique »: « mobiliser une conception pluraliste de l'histoire contre l'étrécissement identitaire qui domine aujourd'hui le débat public » et pour cela "expliquer la France par le monde, écrire l'histoire d'une France qui s'explique avec le monde». Jules Michelet et Lucien Febvre avaient déjà souligné l'intérêt de cette approche. Mais il s'agissait bien d'écrire « une histoire mondiale de la France et non pas une histoire de la France mondiale», non pas de «suivre l'expansion au long cours d'une France mondialisée ». Prendre en compte donc simplement le fait que «la France n'existe pas séparément du monde, [que] le monde n'a jamais la même consistance pour la France ». Le choix d'une collection de dates avait aussi d'après P. Boucheron, un avantage moins immédiatement perceptible pour le lecteur : «L'entrée par les dates s'imposait comme la manière la plus efficace pour déjouer les continuités illusoires du récit traditionnel: elle permet d'évoquer des proximités pour les déplacer ou au contraire de domestiquer 
d'apparentes incongruités ». Cela dit P. Boucheron reconnaissait aussi avec prudence un certain nombre de limites. "Les 146 dates ne forment pas vraiment une chronologie». Elles "valorisent inévitablement une lecture politique et culturelle, négligeant sans doute les évolutions de plus longue durée affectant l'histoire des sociétés dans leur dimension économique mais aussi environnementale ». Mais il osait dire - ce qui est sympathique - que le choix des dates avait été aussi guidé par un " principe de plaisir ». Ces précautions admises, il nous faut bien considérer comment le XIX siècle est abordé dans l'ouvrage.

2 Remarquons d'abord que ce dernier n'est pas individualisé dans les séquences chronologiques du livre qui distinguent d'abord une période qui va de 1795 au milieu $\mathrm{du} \mathrm{XIX}^{\mathrm{e}}$ siècle, sous le titre "Une patrie pour la révolution mondiale ». Puis une autre période intitulée, «La mondialisation à la française » s'étend jusqu'en 1907, suivie de " Modernités dans la tourmente », qui se prolongera jusqu'en 1960. Pour reconstruire en quelque sorte un XIX siècle à partir de l'ouvrage, il faut partir de la date de 1811 («Ce qu'il reste de l'Empire ») et, dépassant la création des Demoiselles d'Avignon par Picasso en 1907 et l'inauguration du Negresco à Nice en 1913 aboutir naturellement à la guerre de 1914. Sur les 146 dates que l'ouvrage présente au total, 24 soit 16,5\% concernent chronologiquement le XIX ${ }^{\mathrm{e}}$ siècle au sens traditionnel de ce terme, ce qui peut paraître raisonnable.

3 Une histoire mondiale de la France ou une histoire de la France mondiale ? Revenons sur cette distinction établie au profit de la première par P. Boucheron. Si l'on suit sa pensée, cela veut dire que la première ne s'attache pas essentiellement à ce qui fait la puissance française dans le monde sur le plan matériel, c'est-à-dire l'extension territoriale notamment coloniale, la puissance économique à travers les investissements à l'étranger, les systèmes d'alliance qui consolident sa position diplomatique, les guerres qu'elle peut mener et qui peuvent modifier son territoire (comme la guerre d'Italie ou celle de 1870) et sa place dans le monde. C'est ainsi que ne sont évoquées en tant que telles, pour le XIX ${ }^{\mathrm{e}}$ siècle, aucune guerre ${ }^{1}$, ni fait important de la conquête coloniale proprement dite. Il est significatif par exemple que la seule allusion à l'Algérie est le projet sans suite de royaume arabe de Napoléon III. Ce que l'ouvrage vise, ce sont plutôt les influences pacifiques. Elles peuvent être le résultat d'initiatives d'ordre scientifique, technique ou culturel (modèle français du Musée, création de l'Alliance française, installation d'une filiale de l'institut Pasteur à Saïgon, reconnaissance internationale de la science française avec les Curie, réalisation remarquable comme celle du canal de Suez, etc.) ou bien résulter d'un impact d'ordre intellectuel, politique ou social (la naissance des idées sociales dans les années 1840, la révolution de 1848, ou la Commune, l'influence de la pensée comtienne au Brésil, le rayonnement mondial d'un lieu de pèlerinage comme Lourdes), ou encore refléter l'adaptation de nouveaux comportements comme l'extension du tourisme aisé sur la Côte d'Azur, ressortir enfin d'intentions humanitaires comme l'appui aux Grecs insurgés en 1825. Le résultat est sans doute de faire apparaître cette histoire mondiale de la France comme un peu idéalisée puisque les initiatives retenues sont toutes positives. Et l'on pourra reprocher au livre de passer sous silence des moments qui relèvent aussi d'une expansion internationale beaucoup moins glorieuse pour la réputation de la France.

4 Partir des dates est certes utile dans une intention pédagogique, mais entraîne de fortes contraintes. Tout d'abord, ces dates sont-elles toutes de la même importance? 
Certaines tout d'abord ne paraissent pas s'imposer. Pourquoi faire une place au discours de Renan sur la nation en 1882 qui n'apparait guère avoir de portée internationale? D'autres n'ont eu qu'un impact différé comme en 1842 la mise au point du plan de la Comédie humaine par Balzac ou en 1883 la création de l'Alliance française. Il est évident que le libre échange en 1860, même s'il n'a duré qu'un temps limité ou la construction du canal de Suez sont des événements majeurs à côté de la référence à la pensée comtienne dans la constitution brésilienne dont on peut se demander si elle a réellement influé dans le long terme sur la politique de ce pays. Cette différence entre les dates a conduit les auteurs à traiter très différemment de leur sujet et toutes les figures ont été utilisées, une monographie sur l'événement lui-même comme dans le cas des Demoiselles d'Avignon, une insertion de la date dans un temps relativement court (comme pour le projet de nation arabe ou les attentats anarchistes), un traitement du sujet sur une assez longue durée pour les expositions universelles, le choléra de 1832 ou le canal de Suez. Parfois l'auteur infléchit son sujet jusqu'à le transformer sensiblement. Ainsi, à propos de la Comédie humaine de Balzac, l'auteur montre avec justesse que la promotion du roman à l'échelle mondiale a été largement préparée par l'écossais Walter Scott.

5 Si l'on essaie maintenant de classer les 24 dates retenues dans des rubriques thématiques, ce qui n'est pas toujours facile car certaines peuvent relever de plusieurs rubriques, on peut établir le classement suivant: Politique : 4 ; Économie : 4 ; Aspects internationaux : 6 (dont colonies : 2); Religion: 1; Société : 2; Culture, science: 7 . Certes, le champ est largement ouvert et un certain équilibre est respecté, mais effectivement les dates retenues privilégient assez nettement l'aspect culturel. En revanche le politique occupe une place assez faible. L'influence internationale de la politique française est certes évoquée à propos de 1848 ou de la Commune, mais d'autres aspects auraient mérité d'être relevés (l'héritage international de la grande Révolution, l'accueil des exilés ne méritaient-ils pas d'être signalés ?). Et, d'autre part, n'aurait-il pas été intéressant de faire apercevoir que d'autres aspects, pourtant très importants pour la France, comme le combat pour la République et plus tard pour la laïcité n'ont pas eu le même impact international bien que celui-ci ait pu malgré tout exister? Ainsi, on aurait mieux saisi que l'insertion internationale de la France n'empêchait pas la persistance d'une forte spécificité française. De même, le champ ici très nettement délimité de la politique internationale ne fait guère apercevoir que la France est alors une des principales puissances mondiales.

6 Notre analyse n'a porté que sur le XIX siècle et ne saurait valoir pour tout l'ouvrage. Au final, l'objectif défini au départ est-il atteint? Oui dans une certaine mesure. Et d'abord sur le plan éditorial. Les contributions, titrées parfois de façon à être attractives ${ }^{2}$ sont toutes intéressantes et agréables à lire. La lisibilité de l'ouvrage est réelle. Le contenu de l'ouvrage remédie-t-il à «l'étrécissement du débat public »? Dans une certaine mesure seulement. Certes, il fait apercevoir différents aspects des interrelations entre la France et le monde. Ces aspects sont-ils les plus déterminants pour l'influence et le rayonnement français dans le monde? Ne boudons pas malgré tout notre plaisir puisque celui-ci était aussi un des objectifs de l'ouvrage. L'entreprise devait être tentée et, malgré les réserves que nous avons mentionnées, reste salutaire. 


\section{NOTES}

1. La guerre de 1870 n'est évoquée qu'à propos de la Commune.

2. Telle « le rayonnement sous X de la science française ", pour le Prix Nobel à Marie Curie ou « Du Zambèze à la Corrèze, une seule langue mondiale », pour la création de l'Alliance française. 